\title{
Hydatid cysts in muscles: clinical manifestations, diagnosis, and management of this atypical presentation
}

\author{
Recep Tekin ${ }^{[1]}$, Alper Avci ${ }^{[2]}$, Rojbin Ceylan Tekin ${ }^{[3]}$, Mehmet Gem ${ }^{[4]}$ and Remzi Cevik ${ }^{[5]}$
}

[1] Department of Clinical Microbiology and Infectious Diseases, Faculty of Medicine, Dicle University, Diyarbakir, Turkey. [2]. Department of Thoracic Surgery, Faculty of Medicine, Cukurova University, Adana, Turkey. [3]. Department of Radiology, Diyarbakır Genesis Hospital, Diyarbakır, Turkey. [4]. Department of Orthopaedic Surgery, Faculty of Medicine, Dicle University, Diyarbakir, Turkey. [5]. Department of Physical Medicine and Rehabilitation, Faculty of Medicine, Dicle University, Diyarbakir, Turkey.

\begin{abstract}
Introduction: Hydatid cysts are rarely detected in muscle tissue (0.7-0.9\%), even in endemic countries. The aim of this study was to present information regarding the clinical manifestations, diagnosis, and management of muscle echinococcosis. Methods: Twenty-two patients with hydatid cysts in the muscle were followed from January 2006 through December 2014. Results: Twenty-four sites of muscle involvement were observed in the 22 patients. Fifteen (68\%) of our patients were women, while seven (32\%) were men. The mean age was $28.1 \pm 15.4$ (6-61) years. The most frequent locations were the thigh (27.2\%) and the paravertebral region (13.6\%). Most patients reported a painless slow-growing mass with normal overlying skin. Most $(90.2 \%)$ cases were treated by surgical excision and fine-needle aspiration. Conclusions: Primary muscle hydatid cyst should be considered in the differential diagnosis in cystic masses of the muscular system without pain and localized enlargement of soft tissue, especially in endemic areas. Hydatid cyst should be investigated using serological tests and imaging modalities. If possible, total surgical excision of hydatid cyst in the muscle should be performed.
\end{abstract}

Keywords: Echinococcosis. Hydatid cyst. Muscle.

\section{INTRODUCTION}

Hydatid cyst disease is an endemic parasitic infection caused by Echinococcus granulosus, and is an important public health issue in Mediterranean countries, the Middle East, Africa, Asia, South America, and Australia ${ }^{(1)}$. The liver and lungs are the two most frequently affected organs in humans, because the swallowed eggs hatch in the intestines and are transported by the portal system to the liver ${ }^{(2)}$. The localization of hydatid cyst in muscle is rare (0.7-0.9\%), even in endemic countries. This primitive and isolated form of echinococcosis can present difficulties in diagnosis and management, particularly for inexperienced clinicians, and it must be correctly differentiated from soft-tissue tumors ${ }^{(3)}$. The aim of this study was to present information regarding the clinical manifestations, diagnosis, and management of atypical muscle echinococcosis. To the best of our knowledge, this study is the most comprehensive case series published in the literature.

Corresponding author: Dr. Recep Tekin. Department of Infections Disease, Faculty of Medicine of Dicle University, Yenişehir 21280 Diyarbakır, Turkey. Phone: +90 41224880 01- 4858

e-mail: receptekin@yahoo.com

Received 17 June 2015

Accepted 18 August 2015

\section{METHODS}

Twenty-two patients who were monitored by different clinics for soft-tissue cystic echinococcosis from January 2006 through December 2014 were included in the study. All patients lived in Turkey, which is an endemic area for hydatid disease. The following medical information was retrospectively collected from patient medical records: epidemiological data, gender, age, occupation, cyst location, clinical examination, clinical presentation, history and physical elements associated with the enlarging mass, laboratory findings, imaging techniques, fine-needle aspiration (FNA), neoadjuvant chemotherapy, therapeutic options with surgery, postoperative adjuvant medical therapy, follow-up period, and recurrences. Results of an immunofluorescence assay (IFA) test to detect echinococcosis antibodies were recorded. For follow-up care, ultrasonography (US) or magnetic resonance imaging (MRI) examination was performed and compared with previous studies to detect new onset or recurrence of hydatid cyst ${ }^{(1)(2)}$. In patients diagnosed with more than one hydatid cyst, only one lesion underwent histopathological examination, and remaining lesions with similar or characteristic radiological features were accepted as hydatid cysts. 


\section{RESULTS}

Twenty-two patients were included in the study. Fifteen $(68 \%)$ of the patients were women, while seven $(32 \%)$ were men, with a mean age of $28.1 \pm 15.4(6-61)$ years. The lower extremities were involved in eight $(36.3 \%)$ patients, with the thigh muscle being the most common location [six (27.2\%) patients], followed by a paravertebral site [three $(13.6 \%)$ patients]. The upper extremities were the rarest location, being involved in only one $(4.5 \%)$ patient. An overview of sociodemographic data, symptoms, clinical signs, and treatment in the 22 patients is given in Table 1 . The main symptom was a painless slow-growing mass with normal overlying skin. Clinically, the gradual emergence of a soft-tissue swelling, lasting for a year on average, was the main reason for consultation. MRI was the most common imaging modality used in the patients in our study (Figure 1). In all patients, the cysts were confined to the muscle and did not affect neurovascular structures or bones, and the diagnosis of muscle echinococcosis was confirmed by histopathological examination. We were able to obtain only seven IFA tests in our patients, and all IFA test results exceeded the $1 / 160$ value. Most of the patients [18 (81.8\%) patients] underwent complete surgical resection (Figure 2).

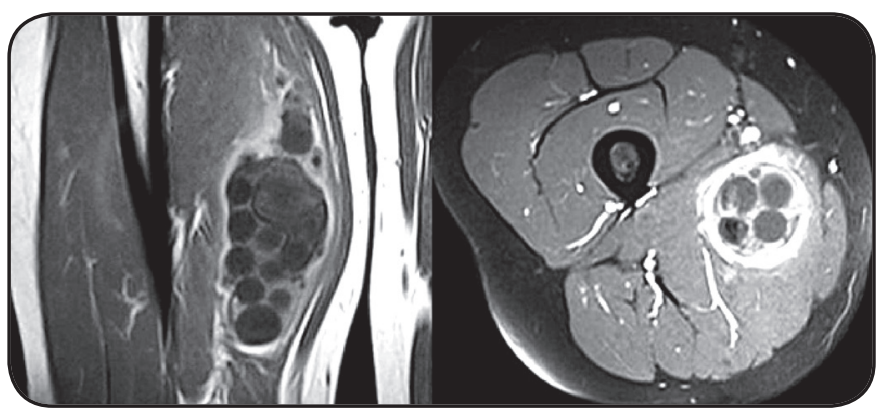

FIGURE 1 - Contrast-enhanced magnetic resonance images of a cystic mass arising from the origin of the right adductor muscle. A hyperintense multicystic lesion with multiple daughter cysts is seen.

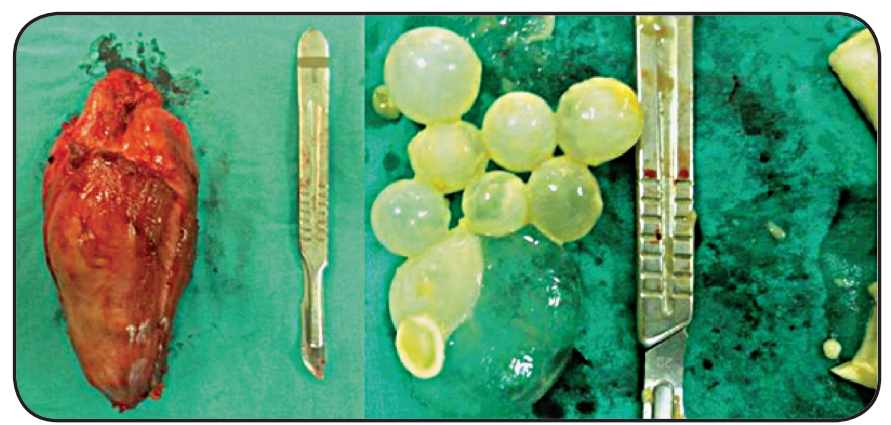

FIGURE 2 - Postoperative view of the mass and macroscopic aspect. The surgical specimen contained multiple small daughter cysts.

\section{DISCUSSION}

Cystic echinococcosis remains a health issue in developing countries because of the lack of strict control programs to prevent the transmission of this infection and problems such as high populations of stray dogs, illegal butchering of animals, and poor public education about disease ${ }^{(4)}$. The liver $(50-70 \%)$ and lungs (20-30\%) are the most frequent organs affected by hydatid cysts. Muscle hydatidosis is rare, possibly because of muscle lactic acid content and muscle contraction, two factors that likely prevent cyst growth in striated muscle. However, parasitic cysts are inclined to grow in the trunk, neck, and legs because of relatively less muscle contraction and more vascularization of these areas. Intramuscular hydatid cysts have been reported in the muscles of the chest wall and in the pectoralis major, sartorius, quadriceps, and gluteus muscles ${ }^{(5)(6)}$. Primary hydatid cyst in muscular tissue has a very low incidence, with only select reports in the literature. Khanna et al. ${ }^{(7)}$ reported 24 unusual hydatid cyst locations among 110 patients with cystic echinococcosis in a period of 23 years. These locations included the spleen, skin, and soft tissue in decreasing order of frequency. Mseddi et al. ${ }^{(8)}$ reported 11 intramuscular hydatid cysts in a period of 17 years.

Clinically, the early stage of muscular hydatid disease is asymptomatic. The symptoms and signs of hydatid cyst disease depend on the involved organ, site of localization, effect on the adjacent tissue, complications after rupture, immunological reactions, and secondary infection. A palpable mass is the most constant clinical finding of hydatid disease affecting soft tissues, and the clinical manifestations are caused by compression of the organ involved ${ }^{(9)}(10)(11)$. Muscle hydatid disease most often manifests as a slow-growing soft-tissue tumor, and can imitate myositis or a calcified hematoma. However, some cysts are revealed by complications such as nerve compression or infection, thereby simulating an acute abscess or a malignant tumor ${ }^{(3)}$. Our patients mostly presented with a painless growing mass in an affected part of the body. However, cysts are sometimes clinically asymptomatic and diagnosed incidentally.

If there is accompanying organ involvement and hydatid serologic testing is positive, local muscle involvement may be diagnosed easily. Otherwise, diagnosis of a primary skeletal muscle hydatid cyst is challenging ${ }^{(12)}$. Eosinophilia is usually not detected in healthy cystic echinococcosis. In our patients, only a single (4.7\%) patient showed a positive finding of eosinophilia with $10 \%$ eosinophils. Although imaging methods have a primary role in diagnosis, there is need for serological verification in complicated cases and postoperative evaluation of the patient and in monitoring for recurrence. There are several serologic methods used in serologic diagnosis of cystic echinococcosis ${ }^{(13)}$, including enzyme-linked immunosorbent assay, western blot testing, and indirect hemagglutination antibody testing; however, these tests remain supportive and inadequate alone for the diagnosis of hydatid cyst ${ }^{(14)}$. Nevertheless, serologic tests can be useful for the differential diagnosis of cystic echinococcosis from neoplasm, abscess, simple cyst, or other masses, and for revealing asymptomatic hydatid cyst carriers and the efficacy of public control programs ${ }^{(15)}$. 


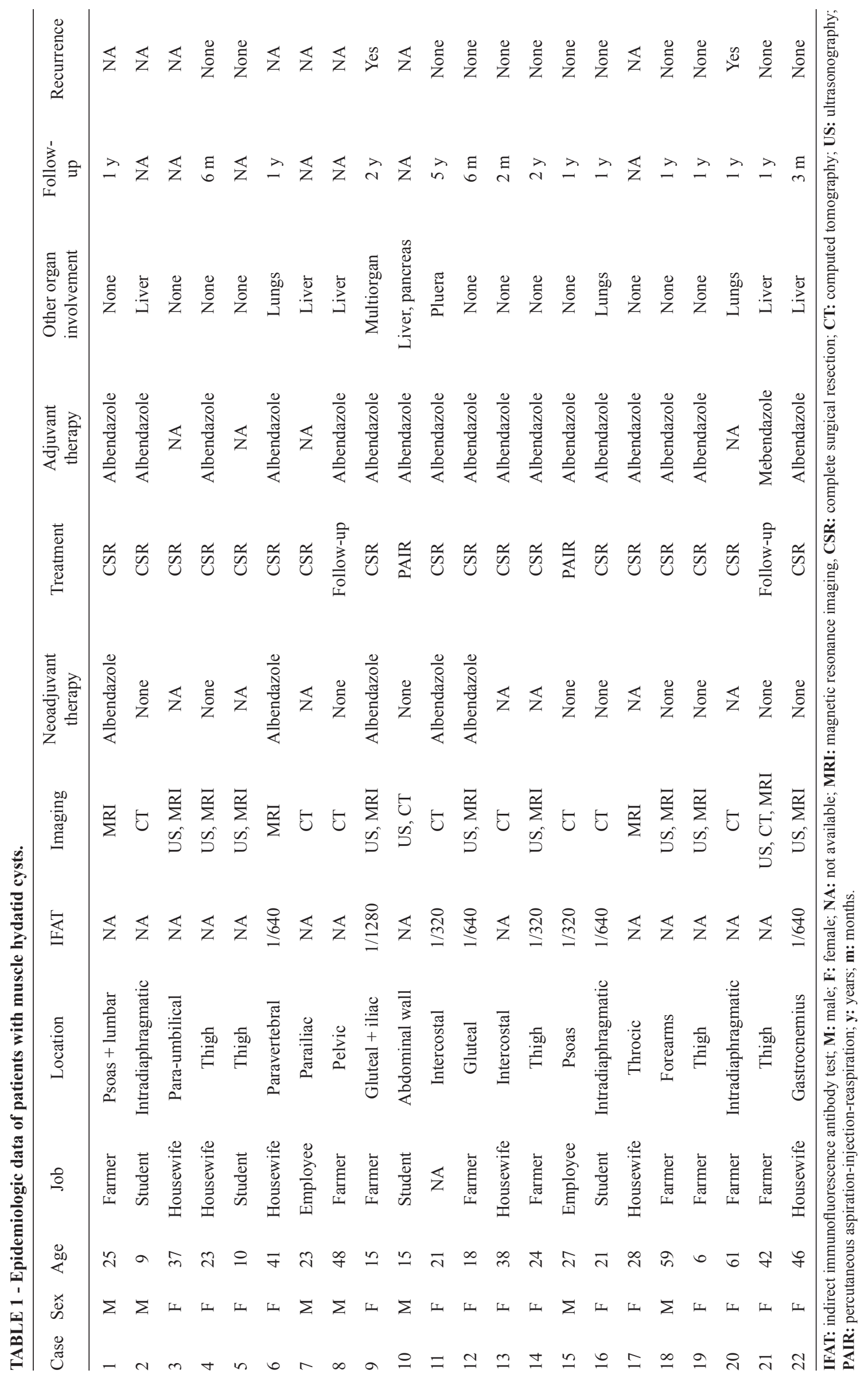


The use of imaging modalities such as US, computed tomography $(\mathrm{CT})$, and MRI in the diagnosis of slow-growing cystic masses in the musculoskeletal system enables accurate visualization of structures and their relation to adjacent tissues. In addition, confirmation of diagnosis of hydatid cyst with these imaging modalities helps to prevent unnecessary cystic puncture and its complications such as local dissemination and anaphylactic shock ${ }^{(9)(16)}$. Danice et al. ${ }^{(17)}$ and Maurmen et al. ${ }^{(18)}$ reported US and CT to be the best methods for diagnosis.

Ultrasonography is a noninvasive, inexpensive, and repeatable imaging modality, which is widely used and accepted in the diagnosis of this disease, and cysts can be classified according to the US criteria of Gharbi ${ }^{(19)}$. In addition, US can be used as a practical guide during interventional procedures. Daughter cysts, detached membranes, and double-line sign are the most characteristic features of hydatid cyst on US ${ }^{(16)}$. Although CT is superior in detecting wall calcifications and bony associations, the appearance of the cyst is rarely typical. The CT findings are variable, and hydatid cyst can appear as a unilocular cyst, a multivesicular lesion, an atypical complex, or a solid lesion. The multivesicular lesion is characteristic of hydatid cyst and reflects multiple daughter cysts within the parent cyst, whereas a solid or complex lesion is the result of inflammatory changes and may mimic a tumor. As it is with the appearance of hydatid cyst on US, the presence of daughter cysts and detached membranes on CT may aid in the diagnosis ${ }^{(9)(20)}$. In addition, bony invasion is critical in soft tissue cysts, and the use of $\mathrm{CT}$ is advised as a part of screening and treatment protocols. In the present cases, MRI and US might not have been useful in the diagnosis. MRI findings of hydatid cysts in the liver are well described, but the diagnosis is more difficult to make in the soft tissue of the musculoskeletal system because the MRI findings are not well described ${ }^{(9)}$. However, MRI is an important imaging modality in the detection and characterization of softtissue masses; therefore, further clarification of characteristic findings with hydatid cyst is needed.

For simple cases of muscle hydatid cyst, the most common form of treatment is surgical removal of the cyst combined with chemotherapy before and after surgery. For inoperable cases, medical treatment and/or percutaneous aspiration-injectionreaspiration (PAIR) are alternative treatment options ${ }^{(21)(22)}$. In our series, 18 patients underwent surgical treatment, two patients underwent PAIR, and two patients received medical treatment only.

Anthelminthic therapy with albendazole or mebendazole is effective against tapeworm disease, and medical treatment alone may be effective in $30-40 \%$ of cases of hydatid disease and may be used for the treatment of patients with inoperable disease $^{(23)}$. Many authors recommend preoperative use of anthelmintics to sterilize the cyst, and reduce the consequences of spillage, and the chances of anaphylaxis and dissemination, at surgery. In addition, postoperative medical treatment reduces the chances of recurrence ${ }^{(23)}$ (24) (25). However, if preoperative prediction of complete removal of an unruptured cyst is not possible, albendazole therapy can be initiated prior to surgery, and postoperative drug therapy is administered in most cases at various dosage regimens ${ }^{(11)}$.
In conclusion, a primary muscle hydatid cyst should be considered in the differential diagnosis in cystic masses of the muscular system without pain and localized enlargement of soft tissue, especially in endemic regions such as our country. Hydatid cysts should be investigated using blood tests and imaging modalities; confirmed cases should be treated with total surgical excision if possible.

\section{CONFLICT OF INTEREST}

The authors declare that there is no conflict of interest.

\section{REFERENCES}

1. Kayaalp C, Dirican A, Aydin C. Primary subcutaneous hydatid cysts: a review of 22 cases. Int J Surg 2011; 9:117-121.

2. Alimehmeti R, Seferi A, Rroji A, Alimehmeti M. Saphenous neuropathy due to large hydatid cyst within long adductor muscle: case report and literature review. J Infect Dev Ctries 2012; 6:531-535.

3. Madhar M, Aitsoultana A, Chafik R, Elhaoury H, Saidi H, Fikry T. Primary hydatid cyst of the thigh: on seven cases. Musculoskelet Surg 2013; 97:77-79.

4. Nath K, Prabhakar G, Nagar RC. Primary hydatid cyst of neck muscles. Indian J Pediatr 2002; 69:997-998.

5. Kocakusak A, Koyuncu A, Arikan S, Senturk O. Primary hydatid cyst of vastus lateralis muscle. Acta Chir Belg 2004; 104:471-472.

6. Versaci A, Scuderi G, Rosato A, Angiò LG, Oliva G, Sfuncia G, et al. Rare localizations ofecchinococcosis: Personal experience. ANZ J Surg 2005; 75:986-991.

7. Khanna AK, Prasanna GV, Khanna R, Khanna A. Unusual sites of hydatid cysts in India. Trop Doct 2005; 5:233-235.

8. Mseddi M, Mtaoumi M, Dahmene J, Ben Hamida R, Siala A, Moula T, et al. Hydatid cysts in muscles: eleven cases. Rev Chir Orthop Reparatrice Appar Mot 2005; 91:267-271.

9. Vasilevska V, Zafirovski G, Kirjas N, Janevska V, Samardziski M, Kostadinova-Kunovska $S$, et al. Imaging diagnosis of musculoskeletal hydatid disease. Prilozi 2007; 28:199-209.

10. Ozgonul A, Sogut O, Cece H, Aydın S, Kürkcüoglu IC. Co-occurrence of diaphragmatic and serratus anterior muscle hydatidosis: an unusual localization. J Emerg Med 2012; 43:219-22.

11. Karimi A, Asadi K, Mohseni F, Hossein Akbar M. Hydatid Cyst of the Biceps Femoris Muscle (a Rare Case in Orthopedic Surgery). Shiraz E Medical J 2011; 12:150-154.

12. Dudkiewicz I, Salai M, Apter S. Hydatid cyst presenting as a soft-tissue thigh mass in a child. Arch Orthop Trauma Surg 1999; 119:474-475.

13. Biava MF, Dao A, Fortier B. Laboratory diagnosis of cystic hydatic disease. World J Surg 2001; 25:10-14.

14. Yazar S, Altıntaş N. Serodiagnosis of cystic echinococcosis in Turkey. Helminthol 2003; 40:9-13.

15. Aydın M, Adıyaman G, Doğruman-Al F, Kuştimur S, Ozkan S. Determination of anti-echinococcus IgG antibodies by ELISA in patients with suspected hydatid cyst. Türkiye Parazitol Derg 2012; 36:61-64.

16. Yucesoy C, Ozturk E, Hekimoglu B. Radiologic findings and percutaneous treatment of a rare giant soft tissue hydatid cyst. JBRBTR 2013; 96:286-289.

17. Danice B. Mirkovic M. İlie M. 20 years experience in the treatment of abdominal echinococcosis (1966-1987). Acta Chir Jugosl 1989; 36:579-582.

18. Maurmen El, Ala Qou ME, Mansouri A, Mokhtari M. Peritoneal echinococcosis, Diagnostic and therapatic problems apropos 34 cases. Chirurgie 1991; 117:854-859. 
19. Gharbi HA, Hassine W, Brauner MW, Dupuch K. Ultrasound examination of hydatid liver. Radiology 1981; 139:459-463.

20. Merkle EM, Schulte M, Vogel J, Tomczak R, Rieber A, Kern P, et al. Musculoskeletal involvement in cystic echinococcosis: Report of eight cases and review of the literature. Am J Roentgenol 1997; 168:1531-1534.

21. Hui M, Tandon A, Prayaga AK, Patnaik S. Isolated musculoskeletal hydatid disease: diagnosis on fine needle aspiration and cell block. J Parasit Dis 2015; 39:332-335.

22. Pathak TK, Roy S, Das S, Achar A, Biswas AK. Solitary hydatid cyst in thigh without any detectable primary site. J Pak Med Assoc 2011; 61:1244-1245.
23. Andalib Aliabady Z, Berenji F, Jamshidi MR. A case report of muscle hydatidosis from iran. Iran J Parasitol 2015; 10:132-135.

24. Tekin R, Kara AF, Tekin RC, Cimen D. Cardiac hydatid cyst case recovered with medical treatment. Anadolu Kardiyol Derg 2011; 11:650-651.

25. Mushtaque M, Mir MF, Malik AA, Arif SH, Khanday SA, Dar RA. Atypical localizations of hydatid disease: experience from a single institute. Niger J Surg 2012; 18:2-7. 